\title{
Preoperative management of children with esophageal atresia: current perspectives
}

This article was published in the following Dove Press journal:

Pediatric Health, Medicine and Therapeutics

18 January 2017

Number of times this article has been viewed

\author{
Filippo Parolini' \\ Anna Lavinia Bulotta' \\ Sonia Battaglia' \\ Daniele Alberti ${ }^{1,2}$ \\ 'Department of Pediatric Surgery, \\ "Spedali Civili" Children's Hospital, \\ "Department of Clinical and \\ Experimental Sciences, University of \\ Brescia, Brescia, Italy
}

\begin{abstract}
Esophageal atresia remains one of the most challenging congenital anomalies of the newborn. In recent years, because of the advances in prenatal diagnosis, neonatal critical care, and surgical procedures, overall outcomes have improved substantially, including for premature children. Nowadays, most of the research is focused on medium- and long-term morbidity, with particular reference to respiratory and gastroesophageal problems; the high frequency of late sequelae in esophageal atresia warrants regular and multidisciplinary checkups throughout adulthood. Surprisingly, there are few studies on the impact of prenatal diagnosis and there is continuing debate over the prenatal and preoperative management of these complex patients. In this review, we analyze the literature surrounding current knowledge on the management of newborns affected by esophageal atresia, focusing on prenatal management and preoperative assessment.
\end{abstract}

Keywords: prenatal diagnosis, esophageal atresia, tracheoesophageal fistula, ultrasound scan, tracheobronchoscopy

\section{Introduction}

With an incidence of 2.43 cases per 10,000 births, esophageal atresia (EA), with or without tracheoesophageal fistula (TEF), remains one of the most challenging congenital anomalies of newborn babies. ${ }^{1,2}$ Due to advances in prenatal diagnosis, neonatal critical care, and surgical procedures, overall outcomes have improved substantially in recent years, including for premature children. ${ }^{2-4}$ Nevertheless, EA is still associated with a life-long risk of complications, even if mortality is currently limited to those cases with associated severe life-threatening anomalies. Many unanswered questions still remain in surgical and clinical arenas. In the latter scenario, a continuing debate over the prenatal and preoperative management of these complex patients is ongoing, and more evidence-based recommendations are impelling. ${ }^{3-5}$ In this review, we analyze the current knowledge on the management of newborns affected by EA, focusing on prenatal and preoperative assessment.

\section{Classification}

The variants of EA have been described using many anatomical classification systems. ${ }^{1,2}$ To avoid ambiguity, the clinician should use a narrative description. Nevertheless, Gross of Boston described the classification system that is most often cited. ${ }^{1}$ According to Gross' classification, the primary types of congenital EA are EA with distal TEF (85\%, Gross C), isolated EA without TEF (8\%, Gross A), TEF without
Correspondence: Filippo Parolini Department of Pediatric Surgery, "Spedali Civili" Children's Hospital, Piazzale Spedali Civili I, 25I23 Brescia BS, Italy Tel +390303996201

Fax +39030399 6154

Email parfil@hotmail.it 
atresia or H-type TEF (4\%, Gross E), EA with proximal TEF (3\%, Gross B), and EA with proximal and distal TEF $(<1 \%$, Gross D) $)^{2}$ (Figure 1).

\section{Antenatal considerations}

\section{Ultrasonographic prenatal diagnosis}

Ultrasonographic prenatal diagnosis of EA remains difficult and challenging, with prenatal detection rates reported as varying from $10 \%$ to $50 \%$ of cases ${ }^{5,6}$ Multicenter studies on prenatal diagnosis are lacking, and only few national registers are currently available (Table 1 ). ${ }^{7-9}$ Traditionally, a small or absent gastric bubble in combination with maternal polyhydramnios have been the features advocated as the most sensitive sign of EA. ${ }^{3}$ Nevertheless, the combination of these two signs gave only modest predictive values, ranging from $44 \%$ to $56 \%{ }^{4,6,10}$ Moreover, in cases of TEF, the passage of amniotic fluid through the fistula fills the stomach and usually prevents the development of polyhydramnios. For this reason, in the study by Spaggiari et al, polyhydramnios and small/ absent stomach bubbles were observed in $100 \%$ of cases of type A EA (pure EA without TEF) and only in $46.3 \%$ of cases with TEF (types B, C, D, and E, according to Gross' classification. ${ }^{1,11}$ Similar results were reported by the 2013 French National Register, in which the prenatal suspicion of type A EA was $86 \%$ and only $12 \%$ in cases with TEF. ${ }^{11}$ Considering that EA with TEF is by far the most common type of EA, this is why the overall prenatal diagnosis of EA remains quite poor. ${ }^{12}$ The sensitivity of prenatal ultrasound (US) was, therefore, significantly higher for those scans performed in tertiary center hospitals compared with those performed in district general hospitals. ${ }^{11-14}$ The combination of a small stomach together with the so-called pouch sign has been reported to be diagnostic for EA in few studies. ${ }^{13-16}$ This direct finding consists of the blind-ending pouch in the fetal neck or mediastinum during fetal swallowing, and it gave excellent predictive values. ${ }^{14}$ Nevertheless, the pouch sign is clearly detectable only after the 26 th week of gestation (Table 2); as hypothesized by Kalache et al, in the first and early second trimester, the fetus is not able to generate sufficient pressure on swallowing to permit dilatation of the blind-ending esophagus. ${ }^{14}$ The localization of the upper pouch may also be significant in predicting the outcomes of the affected fetuses: as reported by Has et al, when the blind esophagus is localized at neck level ("upper pouch sign" or
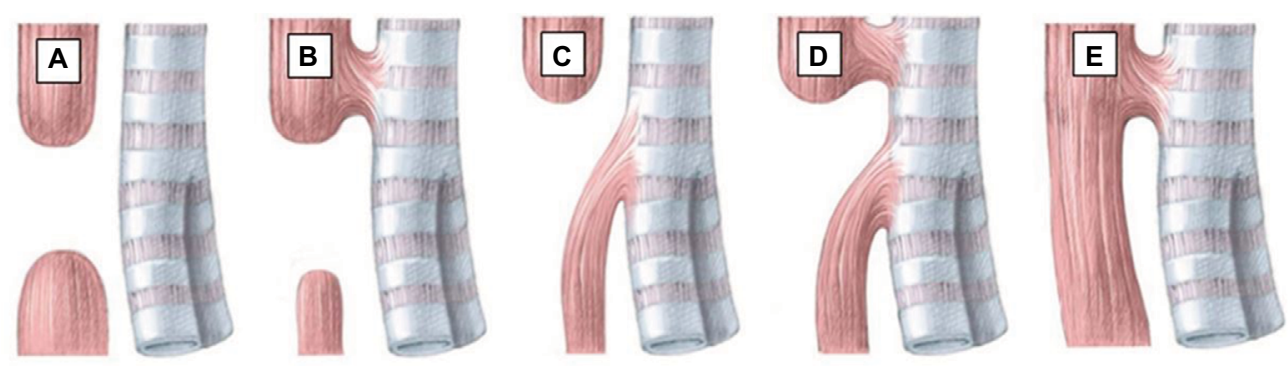

Figure I Anatomical classification of esophageal atresia according to Gross.

Notes: Anatomical classification of EA according to Gross. The primary types of congenital EA are EA with distal TEF ( $85 \%$, Gross C), isolated EA without TEF (8\%, Gross A), TEF without atresia or H-type TEF (4\%, Gross E), EA with proximal TEF (3\%, Gross B), and EA with proximal and distal TEF (<1\%, Gross D).

Abbreviations: EA, esophageal atresia; TEF, tracheoesophageal fistula.

Table I Prenatal diagnosis in recent published data

\begin{tabular}{|c|c|c|c|c|c|}
\hline Author & Setting & Period & $\begin{array}{l}\text { Number } \\
\text { of EA } \\
\text { patients }\end{array}$ & $\begin{array}{l}\text { Prenatal } \\
\text { detection } \\
\text { rate }(\%)\end{array}$ & Note \\
\hline Pini Prato et $\mathrm{al}^{7}$ & Italian National Register & 2012 & 146 & 29.6 & $\begin{array}{l}\text { Prenatal diagnosis significantly more frequent in } \\
\text { Gross' types A and B EA, compared with types C } \\
\text { and D EA }(8 / 10=80 \% \text { vs } 34 / 131=26 \%)\end{array}$ \\
\hline Leoncini et $\mathrm{al}^{8}$ & $\begin{array}{l}\text { Western Australian Register of } \\
\text { Developmental Anomalies (WARDA) }\end{array}$ & 1980-2009 & 260 & 7.7 & $\begin{array}{l}\text { Prenatal detection rate increased during the study } \\
\text { period, with a corresponding decrease in the } \\
\text { proportion of cases diagnosed in the first week } \\
\text { of life }\end{array}$ \\
\hline Garabedian et $\mathrm{al}^{9}$ & French National Register & $2008-2010$ & 408 & 18. I & $\begin{array}{l}\text { The morbidity rate for infants with prenatal } \\
\text { diagnosis is significantly higher than for infants } \\
\text { with postnatal diagnosis, with no difference in } \\
\text { mortality rate }\end{array}$ \\
\hline
\end{tabular}


Table 2 Prenatal findings suspected for EA

\begin{tabular}{|c|c|}
\hline Findings & Notes \\
\hline \multicolumn{2}{|l|}{ Ultrasound } \\
\hline Maternal & High sensibility \\
\hline polyhydramnios & $\begin{array}{l}\text { Low specificity: reported in up to } 10 \% \text { of } \\
\text { pregnancies } \\
\text { After } 26 \text { th week of gestation }\end{array}$ \\
\hline $\begin{array}{l}\text { Absent or small gastric } \\
\text { bubble }\end{array}$ & $\begin{array}{l}\text { In combination with polyhydramnios: modest } \\
\text { predictive values, ranging from } 44 \% \text { to } 56 \% \\
\text { More specific for pure EA (type I) }\end{array}$ \\
\hline \multirow[t]{2}{*}{ "Lower pouch sign" } & $\begin{array}{l}\text { High specificity, but not pathognomonic } \\
\text { for EA }\end{array}$ \\
\hline & $\begin{array}{l}\text { Predictive value for gap between esophageal } \\
\text { pouches } \\
\text { After } 26 \text { th week of gestation }\end{array}$ \\
\hline Regurgitation & After 26th week of gestation \\
\hline "Tracheal print sign" & $\begin{array}{l}\text { The length of the tracheal widening could } \\
\text { estimate the gap between esophageal pouches }\end{array}$ \\
\hline \multicolumn{2}{|c|}{ Magnetic resonance imaging } \\
\hline $\begin{array}{l}\text { Nonvisualization of the } \\
\text { intrathoracic esophagus }\end{array}$ & High specificity \\
\hline $\begin{array}{l}\text { Absent or small gastric } \\
\text { bubble }\end{array}$ & More specific for pure EA (type I) \\
\hline $\begin{array}{l}\text { "Pouch sign" and } \\
\text { distension of the lower } \\
\text { esophagus }\end{array}$ & $\begin{array}{l}\text { When visualized, distension of the lower } \\
\text { esophagus indicates the possible presence of } \\
\text { a tracheoesophageal fistula, while its absence } \\
\text { favors a type I EA }\end{array}$ \\
\hline $\begin{array}{l}\text { Tracheoesophageal } \\
\text { fistula identification }\end{array}$ & High specificity \\
\hline Tracheal bowing & High specificity \\
\hline
\end{tabular}

Abbreviation: EA, esophageal atresia.

"neck sign"), EA is more likely to be associated with long-gap atresia, whereas a mediastinal pouch ("lower pouch sign") is more likely to be associated with distal TEF and short gap atresia. ${ }^{16}$ Nevertheless, Solt et al reported a small series of fetuses with polyhydramnios and a pouch sign, in which the neonatal workup ruled out EA in all cases. ${ }^{17}$ Eventually, Quarello et al reported an indirect sign of EA consisting of a weakness of the posterior wall of the trachea next to the esophageal defect ("tracheal print sign"); in all the six patients in their study, the length of the tracheal widening provides an estimation of the gap between esophageal pouches. ${ }^{18}$

\section{Magnetic resonance imaging (MRI)}

Recently, fetal MRI has been used to identify thoracic and cardiac malformations. ${ }^{19}$ In cases of EA suspicion at prenatal US, increasing evidence suggests that the second-line MRI is required in order to refine the diagnosis. ${ }^{911,20-22}$ The two most sensitive and specific features of EA at MRI are the nonvisualization of the intrathoracic portion of the esophagus and the pouch sign. ${ }^{11}$ The direct visualization of a TEF is pathognomonic, although extremely uncommon. ${ }^{20}$ Nevertheless, according to Hochart et al, in patients in whom a pouch sign is visualized, distension of the lower esophagus indicates the possible presence of a TE fistula, whereas its absence favors type A. ${ }^{21}$ Salomon et al proposed the use of rapid dynamic T2-weighted sequences in order to visualize the swallowing fetus more dynamically ("Fast Imaging Employing SteadyState Acquisition"). ${ }^{22}$ As these dynamic sequences should be repeated as many times as necessary in order to observe sufficient fetal swallowing, MRI is a relatively lengthy examination. ${ }^{21}$ Moreover, the quality of the MRI images is related to the amount of amniotic fluid, which potentially increases fetal motion. Fetal MRI may not be also routinely available in all centers. ${ }^{22}$ Despite these limitations, prenatal MRI could also focus on associated malformations that are reported in up to $50 \%$ of patients with EA, contributing to the overall evaluation through the use of specific sequences, as required, for the evaluation of the nervous system, the chest, or the abdomen. ${ }^{1,21}$

\section{Biochemistry of amniotic fluid}

Only a few studies have evaluated the biochemistry of the amniotic fluid in fetuses with EA. ${ }^{23-27}$ Morin et al first reported that amniotic fluid from pregnancies with fetuses with intestinal obstruction presented diminished microvillar enzyme activities, as compared to normal fetuses. ${ }^{23}$ In subsequent years, abnormal levels of gamma-glutamyl transpeptidase (GGT) in amniotic fluid were reported in some series. ${ }^{24,25}$ In 2011, Czerkiewicz et al proposed the EA index, corresponding to the multiplication of GGT and alpha-fetoprotein, both expressed in multiples of median; by using a threshold of EA index $>3$, a sensibility of $98 \%$ and a specificity of $100 \%$ were found in the diagnosis of EA. ${ }^{24}$ Less encouraging results were reported by Garabedian et al, who with the same EA index observed $90 \%$ of sensibility and $60 \%$ of specificity. ${ }^{25}$ The evidence with regard to routine amniotic fluid analysis in fetuses with EA is poor, including due to the fact that it has only been assessed in cases of polyhydramnios. ${ }^{26}$

\section{The impact of prenatal diagnosis}

There has been little study of the impact of prenatal diagnosis on the prognosis of newborns with EA., ${ }^{1,627-29}$ Garabedian et al, analyzing data from the French National Register for infants with EA born from 2008 to 2010, concluded that patients with prenatal diagnosis have a higher morbidity rate related to the EA type (types A, B, and/or long gap), even if no difference in mortality was found. ${ }^{9}$ Nevertheless, prenatal diagnosis enables the research of associated abnormalities 
that could affect morbidity. ${ }^{27}$ Stringer et al demonstrated a high incidence of other major anomalies, particularly trisomy 18 , in fetuses with suspected EA, and they indicated much poorer outcomes and a higher mortality in these patients, as compared to children with postnatal diagnosis of EA. ${ }^{29}$ According to Fallon et al, when EA is suspected in a woman with polyhydramnios, prenatal management with amniotic fluid reduction should be considered to reduce the risk of preterm labor, thus exposing the infant to prematurity-related morbidity. ${ }^{28}$ Moreover, prenatal diagnosis allows the parents to choose for the birth to take place close to a neonatal surgery unit to avoid the problem of postnatal transfer. ${ }^{9}$ When an antenatal diagnosis of EA is suspected, cesarean section is recommended in the presence of associated anomalies and not to EA itself. In the Italian National Register, vaginal delivery was reported in $18(42 \%)$ patients with prenatal suspicion of EA, with no statistically significant differences between those with and without antenatal diagnosis of EA. ${ }^{7}$

\section{Perinatal considerations Confirmation of the diagnosis}

At birth, a 10-12 French gauge nasogastric (NG) tube should be passed through the mouth into the esophagus for any infant born of a pregnancy complicated by polyhydramnios; failure to pass the NG tube beyond 11 or $12 \mathrm{~cm}$ has been universally recognized as the classical sign of EA. ${ }^{2,6}$ A simple X-ray of the chest and abdomen shows the tip of the catheter halted in the superior mediastinum, while gas in the stomach and intestine signifies the presence of a distal TEF. ${ }^{2}$ Nevertheless, in very rare instances, radiological demonstration of a catheter reaching the stomach does not exclude the diagnosis of EA, as the NG tube may take an alternative route (through the laryngeal inlet, trachea, TEF, and distal esophagus to reach the stomach), which is a rare but well-known scenario. ${ }^{30,31}$

\section{Preoperative care}

The patient should be positioned sitting upright and a sump catheter should be positioned in the upper esophageal pouch to aspirate saliva continuously under low-pressure suction, in order to decrease the risk of pneumonia from aspiration of upper pouch secretions. ${ }^{2,6}$ Broad-spectrum antibiotics (ampicillin and gentamicin) and vitamin $\mathrm{K}$ analog are traditionally administered. ${ }^{2,4}$ Routine endotracheal intubation should be avoided because of the risk of iatrogenic gastric perforation and of increasing respiratory distress, as the abdomen becomes distended from ventilation through the $\mathrm{TEF}^{2,4}$ In these patients, a rectal probe should be inserted to facilitate the evacuation of air and to minimize the intestinal overdistension leading to respiratory impairment.

\section{Associated anomalies}

A careful clinical examination should be conducted in order to rule out associated abnormalities, which are mainly responsible for the medium- and long-term prognosis in these patients. ${ }^{4}$ Their unequal distribution between EA patients is also important from a clinical perspective, as newborns with isolated EA without TEF (Gross A EA) exhibit anomalies in as many as $65 \%$ of cases, compared with a $10 \%$ observed in patients with TEF without atresia (Gross E EA). ${ }^{4,7}$ All patients should have an echocardiogram prior to surgery, in order to identify any structural anomaly of the heart, which is reported in up to $25 \%$ of patients. ${ }^{1,8,9}$ Moreover, preoperative echocardiogram could accurately detect the correct side of the aortic arch: this anomaly, although only reported in $3.6 \%$ of newborns with EA, poses a dilemma in terms of optimal surgical approach, namely the side of the thoracotomy for EA repair. ${ }^{2,32}$ When a prenatal suspicion is evident, the presence of life-threatening anomalies, including Potter's syndrome, cerebral hypoplasia, and chromosomal anomalies (trisomy of chromosomes 13, 14, and 18), should be accurately investigated. ${ }^{1}$

\section{Preoperative assessment}

\section{Contrast esophagram}

Contrast esophagram has been traditionally performed in order to detect the location of the dilated upper esophageal pouch in relation to the thoracic inlet and to detect a proximal TEF. Nevertheless, the need for contrast esophagram is still debated, as it can give false-negative results (when the fistula is occluded by mucus) or false-positive results (when the contrast identifies the tracheobronchial tree, which is more likely to be aspiration through the larynx rather than through a proximal TEF). ${ }^{2,33}$ Moreover, this procedure involves radiation hazards and may be associated with complications, including aspiration pneumonia. ${ }^{33}$

\section{US scan, computed tomographic (CT) scan, and MRI} Increasing evidence suggests that US scans are a useful noninvasive tool for the diagnostic assessment of newborns with EA in order to outline the tracheoesophageal anatomy, ${ }^{34,35}$ Recently, CT scans and MRIs have been proposed for newborns with EA, to identify the position of the TE fistula and anomalies of the aortic arch. ${ }^{36,37}$ Nevertheless, experience with these diagnostic tools in the preoperative assessment of newborns with EA is very limited, also for concerns regarding 
neonatal transportation to the radiology department, the need for sedation, and CT-related radiation hazards. ${ }^{37}$ Moreover, the routine use of preoperative CT scans or MRIs in these patients is controversial, as the limited information acquired that may contribute to modifying the surgical plan can be easily obtained by preoperative tracheobronchoscopy (TBS) or intraoperatively. ${ }^{38}$ The only exception is represented by the extremely small subset of patients with preoperative diagnosis of right-side aortic arch and long-gap EA, in whom preoperative MRI is recommended in order to define the most appropriate surgical approach. ${ }^{32}$

\section{TBS}

In recent years, preoperative TBS has received increasing attention in the evaluation of the presence of proximal TEF. ${ }^{33,38-40}$ However, a recent European survey demonstrated that only $43 \%$ of the respondent pediatric surgeons surveyed routinely perform preoperative TBS before EA repair. ${ }^{41}$ Beyond confirming the presence and location of most commonly observed lower pouch TEF, TBS enables the evaluation of vocal cord motility, to assess the presence of other specific foregut-associated anomalies (tracheomalacia, tracheal clefts, and so on), and the preoperative determination of the gap between esophageal pouches. Moreover, TBS allows the detection of rare upper pouch TEF, which could be unnoticed during surgery. Therefore, today, endoscopic assessment is an essential part of surgical planning. ${ }^{38,39}$

\section{Assessment of the gap}

Accurate preoperative determination of the gap between esophageal pouches is mandatory in order to define the most appropriate surgical plan. ${ }^{1,432,42}$ Gap measurement has been largely reported in detail and should be performed at the time of preoperative TBS. ${ }^{42,43}$ When a distal TEF is evident, a rigid catheter is inserted into the upper esophageal pouch, while the tip of the bronchoscope is positioned at the level of tracheal opening of distal fistula. Chest fluoroscopy demonstrates the distance between them, which is highly representative of the gap length. ${ }^{32,42}$ Bagolan et al reported that a standardized and reproducible preoperative protocol of gap measurement enhanced the possibility of preserving the native esophagus in cases of long-gap EA. ${ }^{42}$

\section{Future perspectives on prenatal and preoperative assessment of patients with EA}

EA remains one of the most challenging disorders in newborns and, although improvements have been made in the diagnosis and treatment of these patients over the years, much remains to be understood. Nowadays, research is focused on medium- and long-term morbidity, with particular reference to respiratory and gastroesophageal problems; the high frequency of late sequelae in EA warrants regular and multidisciplinary checkups throughout adulthood. Surprisingly, there are few studies on the impact of prenatal diagnosis. An accurate prenatal diagnosis is necessary in order to offer an early multidisciplinary prenatal counseling and to allow parents to choose for the birth to take place close to a highvolume tertiary-level center to offer the best of care and to avoid the problem of postnatal transfer. Unfortunately, despite recent advancements in prenatal diagnosis, the suspicion of EA is stably reported in $<50 \%$ of cases of EA, with higher rate reserved only for uncommon EA variants (types $\mathrm{A}$ and B). Efforts should be made in order to find new signs alternative when maternal polyhydramnios is absent. Increasing evidence suggests that in case of ultrasonographic suspicion of EA, second-level MRI (including real-time MRI with cine mode) should be offered to parents. Moreover, in cases of pregnancy with polyhydramnios and suspected EA, amniotic fluid reduction should be considered to reduce the risk of preterm labor, thus exposing the infant to prematurity-related morbidity. The type of delivery (cesarean section vs vaginal delivery), according to associated anomalies, should be considered to plan appropriate perinatal care with the prompt availability of the multidisciplinary team (obstetrician, neonatologist, radiologist, neonatal surgeon, and neonatal anesthetist). At birth, an early and accurate preoperative diagnosis including TBS and, in selected case, magnetic resonance imaging is necessary to detect anatomical details such as the side of aortic arch and "long-gap" EA, in order to plan the more appropriate surgical approach before surgical repair. An early and accurate evaluation of associated anomalies, which are mainly responsible for the medium- and long-term prognosis in these patients, is also mandatory.

\section{Author contributions}

FP, ALB, SB, and DA conceptualized and designed the study, designed the data collection instruments, drafted the initial manuscript, reviewed and revised the manuscript, and approved the final manuscript as submitted. Authors declare that this manuscript has never been published and it would not be submitted to any other journal while under consideration for publication in your journal.

\section{Disclosure}

The authors report no conflicts of interest in this work. 


\section{References}

1. Pedersen RN, Calzolari E, Husby S, Garne E. Oesophageal atresia: prevalence, prenatal diagnosis and associated anomalies in 23 European regions. Arch Dis Child. 2012;97(3):227-232.

2. Spitz L. Oesophageal atresia. Orphanet J Rare Dis. 2007;2:24.

3. Sulkowski JP, Deans KJ, Asti L, Mattei P, Minneci PC. Using the Pediatric Health Information System to study rare congenital pediatric surgical diseases: development of a cohort of esophageal atresia patients. J Pediatr Surg. 2013;48(9):1850-1855.

4. Pinheiro PF, Simões e Silva AC, Pereira RM. Current knowledge on esophageal atresia. World J Gastroenterol. 2012;18(28):3662-3672.

5. Brantberg A, Blaas H-GK, Haugen SE, Eik-Nes SH. Esophageal obstruction-prenatal detection rate and outcome. Ultrasound Obstet Gynecol. 2007;30(2):180-187.

6. Houben $\mathrm{CH}$, Curry JI. Current status of prenatal diagnosis, operative management and outcome of esophageal atresia/tracheo-esophageal fistula. Prenat Diagn. 2008;28:667-675.

7. Pini Prato A, Carlucci M, Bagolan P, et al. A cross-sectional nationwide survey on esophageal atresia and tracheoesophageal fistula. J Pediatr Surg. 2015;50(9):1441-1456.

8. Leoncini E, Bower C, Nassar N. Oesophageal atresia and tracheooesophageal fistula in Western Australia: prevalence and trends. J Paediatr Child Health. 2015;51(10):1023-1029.

9. Garabedian C, Sfeir R, Langlois C, et al. Does prenatal diagnosis modify neonatal treatment and early outcome of children with esophageal atresia? Am J Obstet Gynecol. 2015; 212(3):340.e1-340.e7.

10. Sfeir R, Bonnard A, Khen-Dunlop N, et al. Esophageal atresia: data from a national cohort. J Pediatr Surg. 2013;48(8):1664-1669.

11. Spaggiari E, Faure G, Rousseau V, Sonigo P, Millischer-Bellaiche AE et al. Performance of prenatal diagnosis in esophageal atresia. Prenat Diagn. 2015;35(9):888-893.

12. Holland AJ, Fitzgerald DA. Oesophageal atresia and tracheo-oesophageal fistula: current management strategies and complications. Paediatr Respir Rev. 2010;11(2):100-106.

13. Kalache KD, Chaoui R, Mau H, Bollmann R. The upper neck pouch sign: a prenatal sonographic marker for esophageal atresia. Ultrasound Obstet Gynecol. 1998;11(2):138-140.

14. Kalache KD, Wauer R, Mau H, Chaoui R, Bollmann R. Prognostic significance of the pouch sign in fetuses with prenatally diagnosed esophageal atresia. Am J Obstet Gynecol. 2000;182(4):978-981.

15. Satoh S, Takashima T, Takeuchi H, Koyanagi T, Nakano H. Antenatal sonographic detection of the proximal esophageal segment: specific evidence for congenital congenital esophageal atresia. J Clin Ultrasound. 1995;23(7):419-423.

16. Has R, Günay S. Upper neck pouch sign in prenatal diagnosis of esophageal atresia. Arch Gynecol Obstet. 2004;270(1):56-58.

17. Solt I, Rotmensch S, Bronshtein M. The esophageal 'pouch sign': a benign transient finding. Prenat Diagn. 2010;30(9):845-848.

18. Quarello E, Saada J, Desbriere R, Rousseau V, De Lagausie P, Benachi A. Prenatal diagnosis and evaluation of defect length in esophageal atresia using direct and indirect (tracheal print) signs. Ultrasound Obstet Gynecol. 2011;38(2):225-228.

19. Levine D, Barnewolt CE, Mehta TS, Trop I, Estroff J, Wong G. Fetal thoracic abnormalities: MR imaging. Radiology. 2003;228(2):379-388.

20. Langer JC, Hussain H, Khan A et al. Prenatal diagnosis of esophageal atresia using sonography and magnetic resonance imaging. $J$ Pediatr Surg. 2001;36(5):804-807.

21. Hochart V, Verpillat P, Langlois C, et al. The contribution of fetal MR imaging to the assessment of oesophageal atresia. Eur Radiol. 2015;25(2):306-314.

22. Salomon LJ, Sonigo P, Ou P, Ville Y, Brunelle F. Real-time fetal magnetic resonance imaging for the dynamic visualization of the pouch in esophageal atresia. Ultrasound Obstet Gynecol. 2009 ;34(4):471-474.
23. Morin PR, Melançon SB, Dallaire L, Potier M. Prenatal detection of intestinal obstructions, aneuploidy syndromes, and cystic fibrosis by microvillar enzyme assays (disaccharidases, alkaline phosphatase, and glutamyltransferase) in amniotic fluid. Am J Med Genet. 1987; 26(2):405-415.

24. Czerkiewicz I, Dreux S, Beckmezian A, et al. Biochemical amniotic fluid pattern for prenatal diagnosis of esophageal atresia. Pediatr Res. 2011;70(2):199-202.

25. Garabedian C, Verpillat P, Czerkiewicz I, et al. Does a combination of ultrasound, MRI, and biochemical amniotic fluid analysis improve prenatal diagnosis of esophageal atresia? Prenat Diagn. 2014;34(9):839-842.

26. Muller C, Czerkiewicz I, Guimiot F, et al. Specific biochemical amniotic fluid pattern of fetal isolated esophageal atresia. Pediatr Res. 2013;74(5):601-605.

27. Eyheremendy E, Pfister M. Antenatal real-time diagnosis of esophageal atresias. J Clin Ultrasound. 1983;11(7):395-397.

28. Fallon SC, Ethun CG, Olutoye OO, et al. Comparing characteristics and outcomes in infants with prenatal and postnatal diagnosis of esophageal atresia. J Surg Res. 2014;190(1):242-245.

29. Stringer MD, McKenna KM, Goldstein RB, Filly RA, Adzick NS, Harrison MR. Prenatal diagnosis of esophageal atresia. J Pediatr Surg. 1995;30(9):1258-1263.

30. Kuti K, Patel R, Chapman S, Jawaheer G. A rare pitfall in the diagnosis of oesophageal atresia. Pediatr Radiol. 2013;43(8):902-904.

31. Kumar M, Thomas N. Appearances are deceptive - passing a nasogastric tube does not always rule out oesophageal atresia. J Clin Diagn Res. 2016;10(4):SD01-SD02.

32. Parolini F, Armellini A, Boroni G, Bagolan P, Alberti D. The management of newborns with esophageal atresia and right aortic arch: a systematic review or still unsolved problem. J Pediatr Surg. 2016;51(2):304-309.

33. Parolini F, Boroni G, Stefini S, et al. Role of preoperative tracheobronchoscopy in newborns with esophageal atresia: a review. World $J$ Gastrointest Endosc. 2014;6(10):482-487.

34. Su P, Yuan Y, Zhang Z, Huang Y, Wang W. Application of high-frequency ultrasound in esophageal atresia with distal fistula. Dis Esophagus. 2014;2794):325-329.

35. Gassner I, Geley TE. Sonographic evaluation of oesophageal atresia and tracheo-oesophageal fistula. Pediatr Radiol. 2005;35(2): 159-164.

36. Fitoz S, Atasoy C, Yagmurlu A, Akyar S, Erden A, Dindar H. Three-dimensional CT of congenital esophageal atresia and distal tracheoesophageal fistula in neonates: preliminary results. AJR Am J Roentgenol. 2000;175(5):1403-1407.

37. Garge S, Rao KL, Bawa M. The role of preoperative CT scan in patients with tracheoesophageal fistula: a review. J Pediatr Surg. 2013;48(9):1966-1971.

38. Atzori P, Iacobelli BD, Bottero S, et al. Preoperative tracheobronchoscopy in newborns with esophageal atresia: does it matter? J Pediatr Surg. 2006;41(6):1054-1057.

39. Parolini F, Morandi A, Macchini F, et al. Esophageal atresia with proximal tracheoesophageal fistula: a missed diagnosis. J Pediatr Surg. 2013;48(6): e13-e17.

40. Shoshany G, Vatzian A, Ilivitzki A, Smolkin T, Hakim F, Makhoul IR. Near-missed upper tracheoesophageal fistula in esophageal atresia. Eur J Pediatr. 2009;168(10):1281-1284.

41. Zani A, Eaton S, Hoellwarth ME, et al. International survey on the management of esophageal atresia. Eur J Pediatr Surg. 2013;24(1):3-9.

42. Bagolan $P$, Valfrè L, Morini F, Conforti A. Long-gap esophageal atresia: traction-growth and anastomosis - before and beyond. Dis Esophagus. 2013;26(4):372-379.

43. Chan KL, Saing H. Combined flexible endoscopy and fluoroscopy in the assessment of the gap between the two esophageal pouches in esophageal atresia without fistula. J Pediatr Surg. 1995;30(5):668-670. 
Pediatric Health, Medicine and Therapeutics is an international, peerreviewed, open access journal publishing original research, reports, editorials, reviews and commentaries. All aspects of health maintenance, preventative measures and disease treatment interventions are addressed within the journal. Practitioners from all disciplines are invited to submit their work as well as healthcare researchers and patient support groups. The manuscript management system is completely online and includes a very quick and fair peer-review system. Visit http://www.dovepress.com/ testimonials.php to read real quotes from published authors.

Submit your manuscript here: http://www.dovepress.com/pediatric-health-medicine-and-therapeutics-journal 